\title{
Confirmation de l'existence en Tunisie de Sergentomyia antennata (Newstead, 1912) (Diptera-Psychodidae)
}

\author{
par J.-A. RIOUX *, J. PERIERES *, R. KILLICK-KENDRICK * * \\ et M. MAISTRE * \\ (Collaboration technique: N. BAYAR) \\ * Laboratoire d'Ecologie médicale et Pathologie parasitaire ( $P^{r}$ J.-A. Rioux), \\ Faculté de Médecine, F 34000 Montpellier \\ ** Department of Zoology and Applied Entomology, \\ Imperial College, London SW 72 AZ England, \\ Institut Pasteur de Tunis, Tunisie
}

\section{Résumé.}

La découverte à Gafsa et à Matmata de plusieurs individus $\sigma^{0}$ et $\$$ de Sergentomyia antennata (Newstead, 1912) permet de confirmer l'existence de cette espèce dans le Sud tunisien.

\section{Summary.}

Confirmation of the presence of Sergentomyia antennata (Newstead, 1912) (Diptera-Psychodidae) in Tunisia.

The discovery at Gafsa and Matmata of several $\delta^{-1}$ and $q$ specimens of Sergentomyia antennata (Newstead, 1912) confirms the presence of this species in southern Tunisia.

Accepté le 20 mars 1978. 
La seule récolte de Sergentomyia antennata, mentionnée jusqu’à présent en Tunisie, est à mettre à l'actif de M. Langeron (1). Publiée en 1921 sous le binôme de Phlebotomus minutus Rondani, 1843, puis reprise par F. Larrousse (1921) sous celui de Phlebotomus africanus Newstead, 1912, cette espèce est à nouveau citée par L. Parrot et J. Clastrier (1944) sous celui de Phlebotomus signatipennis Newstead, 1920. Un an auparavant, L. Parrot avait en effet dénoncé l'erreur, entretenue depuis le début du siècle, sur la signification du trinôme Phlebotomus minutus africanus: sous ce dernier vocable on avait en réalité confondu deux autres espèces: Phlebotomus minutus parroti Adler et Theodor, 1927, et Phlebotomus signatipennis. En 1949, R. Kirk et D.-J. Lewis considéraient à leur tour Phlebotomus signatipennis comme une simple variété de Phlebotomus antennatus Newstead, 1912. Quoi qu'il en soit, la récente découverte de Sergentomyia antennata lors d'une mission dans les gouvernorats de Gafsa et de Médenine confirme l'existence de ce taxon en Tunisie du Sud et étend ipso facto sa répartition à l'ensemble des pays du Maghreb (2).

\section{Observation}

Le 27 août 1977, nous plaçons une série de pièges lumineux adhésifs dans les dépendances d'une habitation de la palmeraie de Gafsa. Parallèlement, nous réalisons un prélèvement extemporané à l'aspirateur-nasse. La détermination des captures donne les résultats suivants :

Phlebotomus papatasi (Scopoli, 1786) ....................... $6 \delta^{2}$

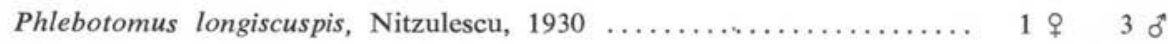

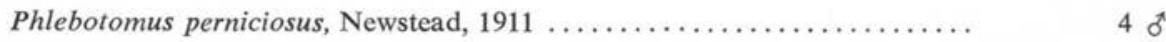

Sergentomyia antennata (Newstead, 1912) ..................... $59 q 155 \delta$

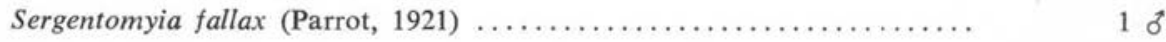

Sergentomyia minuta parroti (Adler et Theodor, 1927) $\ldots \ldots \ldots \ldots \ldots .2 \% \quad 3 \delta$

Le 28 août 1977, au village troglodyte de Matmata, nous effectuons une capture manuelle dans une grotte aménagée en bergerie. Nous identifions les espèces suivantes:

Phlebotomus papatasi (Scopoli, 1786) ........................ $3 q 10 \delta$

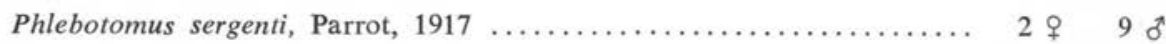

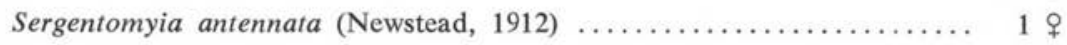

Sergentomyia fallax (Parrot, 1921) ......................... 1 의

Sergentomyia minuta parroti (Adler et Theodor, 1927) ............. 1 \%

(1) Grâce à l'amabilité de notre maître J. Callot, nous avons pu examiner une préparation sur lame (montée au Baume) des récoltes de M. Langeron. L'étiquette porte la méntion: Phlebotomus minutus africanus, Bordj de Tamerza, 1919. Il s'agit en réalité de Sergentomyia antennata $\delta^{\lambda}$ (2 exemplaires) et Sergentomyia minuta ơ (1 exemplaire).

(2) D'après L. Parrot (1942), les expériences sur la transmission de Leishmania tarentolae au Gecko, Tarentola mauritanica, auraient été conduites sur Sergentomyia antennata et non sur Sergentomyia minuta parroti. 


\section{Rappel systématique}

Au plan supraspécifique, Sergentomyia antennata, tant $\sigma^{*}$ que $q$ se sépare difficilement des autres représentants du sous-genre Sergentomyia.

LA FEMELLE possède des spermathèques tubulaires, identiques à celles de Sergentomyia fallax (Parrot, 1921) et de Sergentomyia minuta parroti. La distinction est apportée par la forme des armatures cibariale et pharyngienne. Chez Sergentomyia minuta parroti, les dents cibariales sont nombreuses $(\mathrm{m} \cong 70)$, disposées sur une ligne horizontale, et doublées d'une tache ovalaire sans prolongement antérieur. Chez Sergentomyia antennata, ces mêmes dents sont peu nombreuses $(m \cong 25)$, rangées sur une ligne fortement concave vers l'arrière et doublées par une tache en forme de «casque à pointe». La distinction de Sergentomyia antennata et de Sergentomyia fallax est plus délicate: le caractère le plus intéressant est apporté par la structure de la plaque d'épines pharyngiennes relativement étroite et régulièrement arrondie à la partie postérieure chez Sergentomyia antennata, large et nettement cordiforme chez Sergentomyia fallax.

LE MÂLE présente un pénis terminé en pointe mousse, assez semblable à celui de Sergentomyia minuta parroti et surtout à celui de Sergentomyia fallax (fig. 1). En fait, les caractères discriminants sont à rechercher au niveau du style : chez Sergentomyia antennata, la soie non caduque est implantée en position subapicale et non pas médiane comme chez Sergentomyia minuta parroti. Quant à Sergentomyia fallax, dont la soie non caduque est également implantée en position terminale, il se distingue de Sergentomyia antennata par l'étroitesse remarquable de son style (fig. 2).
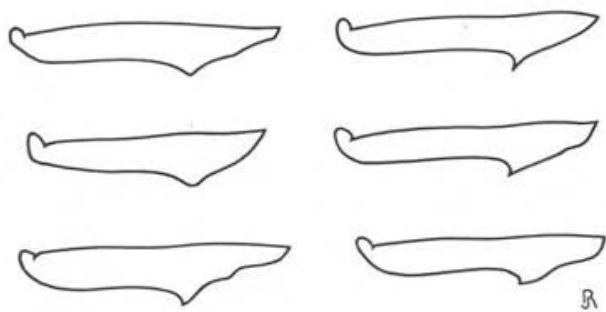

Fig. 1. Sergentomyia antennata (Newstead, 1912). Valves péniennes.

Fig. 2. Sergentomyia antennata (Newstead, 1912). Hypopygium $\delta$.

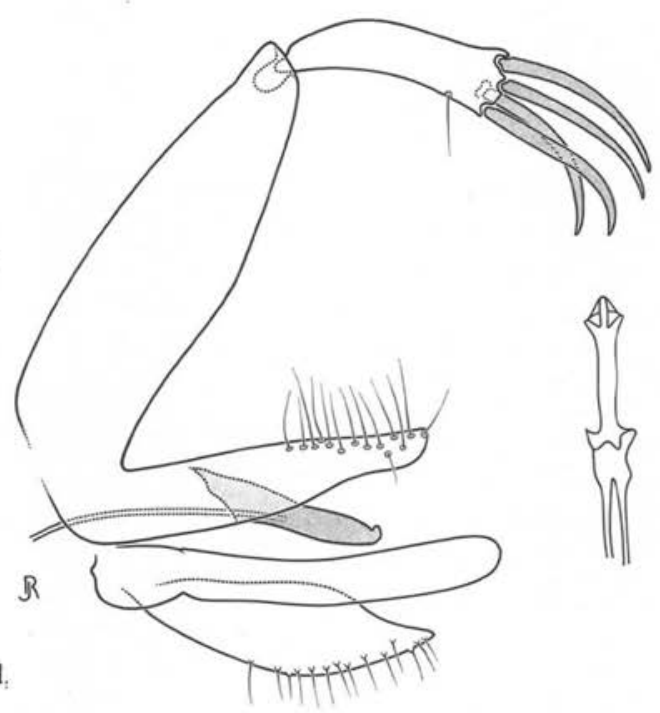


Au plan infraspécifique, rappelons que Sergentomyia antennata avait été considéré jusqu'à ces dernières années comme un complexe systématique groupant au moins trois taxons (antennata, cincta et signatipennis), distingués d'après le nombre de dents du cibarium. Une étude biométrique, réalisée en 1974 sur un important lot provenant du Sud marocain, nous avait permis de discuter et de réfuter partiellement la thèse pluraliste (N. Léger et coll., 1974). La comparaison de deux populations marocaine et tunisienne apporte aujourd'hui un nouvel argument (fig. 3) : les moyennes des distributions sont significativement différentes (à $5 \%$ par le test de l'écart réduit).

Sergentomyia antennata (NEWSTEAD, 1912)

ARMATURE CIBARIALE

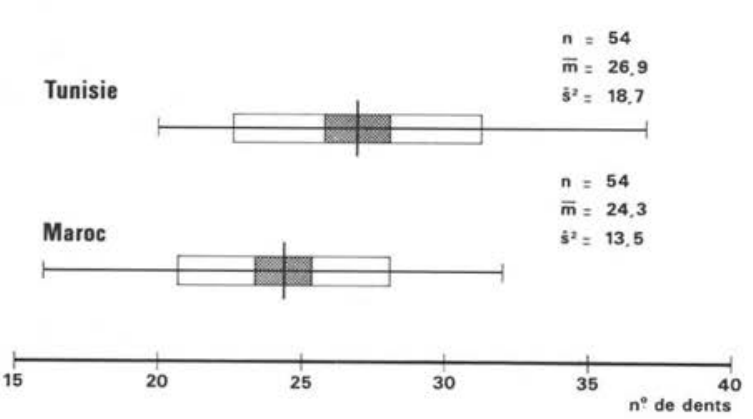

Fig. 3. Distribution de la denture cibariale sur deux échantillons, tunisien et marocain, de Sergentomyia antennata (Newstead, 1912).

Trait vertical: moyenne de l'échantillon; rectangle hachuré : intervalle de confiance de la moyenne; rectangle blanc: écart type de l'échantillon; ligne horizontale : extrêmes observés.

En définitive, le nombre des dents cibariales semble varier sensiblement d'une région à l'autre, peut-être sous la forme d'un cline comme dans le cas de Sergentomyia minuta (J.-A. Rioux et coll., 1975). De telles observations doivent inciter à la prudence. Dans le cas particulier, elles nous amènent une fois de plus à n'utiliser que le seul binôme Sergentomyia antennata pour désigner l'ensemble des populations observées jusqu'à ce jour au Maghreb. Quoi qu'il en soit, après un hiatus d'un demi-siècle, la présence de Sergentomyia antennata en Tunisie est donc confirmée. La liste des Phlébotomes de ce pays s'établit dès lors comme suit :

Phlebotomus (Phlebotomus) papatasi (Scopoli, 1786).

Phlebotomus (Paraphlebotomus) alexandri, Sinton, 1928.

Phlebotomus (Paraphlebotomus) chabaudi, Croset, Rioux et Abonnenc, 1971.

Phlebotomus (Paraphlebotomus) sergenti, Parrot, 1917.

Phlebotomus (Larroussius) ariasi, Tonnoir, 1921.

Phlebotomus (Larroussius) chadlii, Rioux, Juminer et Gibily, 1966. 
Phlebotomus (Larroussius) langeroni, Nitzulescu, 1930.

Phlebotomus (Larroussius) longicuspis, Nitzulescu, 1930.

Phlebotomus (Larroussius) perfiliewi, Parrot, 1939.

Phlebotomus (Larroussius) perniciosus, Newstead, 1911.

Sergentomyia (Sergentomyia) antennata (Newstead, 1912).

Sergentomyia (Sergentomyia) fallax (Parrot, 1921).

Sergentomyia (Sergentomyia) minuta parroti (Adler et Theodor, 1927).

Sergentomyia (Grassomyia) dreyfussi (Parrot, 1935).

Sergentomyia (Sintonius) christophersi (Sinton, 1927).

\section{Bibliographie}

Adler S., Théodor O. (1927): On a collection of Phlebotomus sp. of the minutus group. Ann. trop. Med. Parasit., 21, 61-68.

Kirk R., Lewis D. J. (1949): Taxonomy of the ethiopian sandflies (Phlebotomus). IV. P. bedfor:li Newstead and P. antennatus Newstead. Ann. trop. Med. Parasitol., 43, 333-336.

Langeron M. (1921) : Deuxième mission parasitologique en Tunisie. Tamerza (sept.-oct. 1919). Arch. Inst. Pasteur Afr. N., 1, 347-382.

Larrousse F. (1921): Etude systématique et médicale des Phlébotomes. Thèse Médecine, Paris, 103 p.

Léger N., Rioux J.-A., Croset H., Cadi-Soussi M., Ben Mansour N. (1974): Le «complexe» Sergentomyia (Sergentomyia) antennata (Newstead, 1912). Ann. Parasitol. hum. comp., 49, $577-591$.

Parrot L. (1942) : Notes sur les Phlébotomes. XXXIX. A propos de deux Prophlebotomus d'Algérie • Phlebotomus minutus var. Signatipennis et Phlebotomus fallax. Arch. Inst. Pasteur Algérie, $20,322-335$.

Parrot L. (1943): Notes sur les Phlébotomes. XL. Sur Phlebotomus (Prophlebotomus) minutus Rondani et sa variété parroti Adler et Theodor. Arch. Inst. Pasteur Algérie, 21, 38-47.

Parrot L., Clastrier J. (1944) : Notes sur les Phlébotomes. XLV. Présence de Phlebotomus perfuliewi en Tunisie. Arch. Inst. Pasteur Algérie, 22, 60-62.

Rioux J.-A., Croset H., Léger N., Maistre M. (1975) : Remarques sur la taxonomie infra-spécifique de Sergentomyia minuta, Sergentomyia africana et Sergentomyia antennata. Ann. Parasitol. hum, comp., 50, 635-641. 